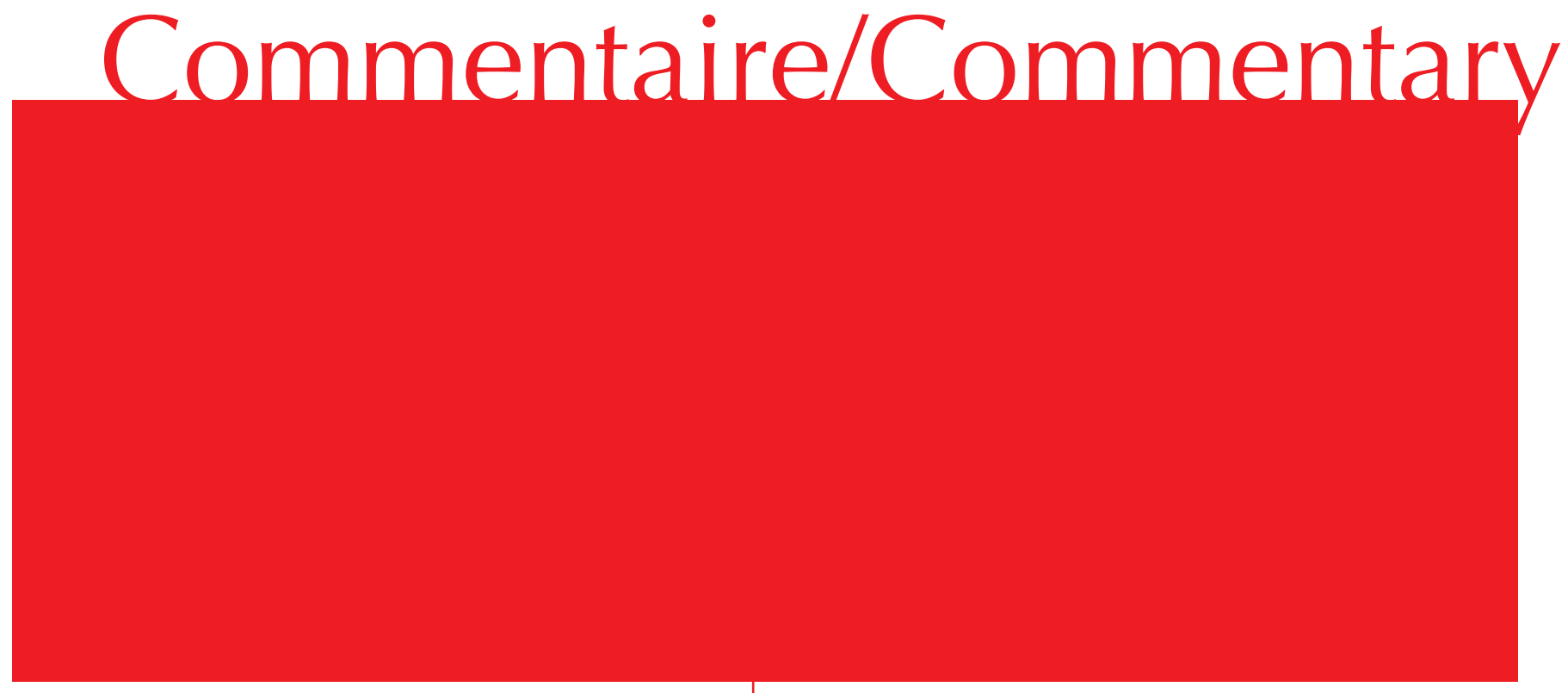

\section{Commentaire de lecture du livre Health Advocacy Inc.}

\section{STÉPHANIE COLLINS}

Health Adocacy Inc. de Sharon Batt[1] explore les liens entre les associations canadiennes de patientes qui font la promotion de la lutte contre le cancer du sein et l'industrie pharmaceutique. À travers une documentation de ces alliances, notamment le financement des associations par le Big Pharma, I'objectif principal de l'auteure est celui de reconstituer le processus de tensions qui s'est opéré à I'intérieur des associations canadiennes de femmes engagées dans la lutte contre le cancer du sein suite au financement de l'industrie. Dans cette perspective, l'auteure veut mettre en lumière les discours et les systèmes de savoir qui soustendent les différentes positions des acteurs impliqués.
Pour ce faire, Batt examine les liens entre l'État, l'industrie pharmaceutique et les associations ainsi que les conditions qui ont modulé leur financement. La problématique est donc éclairée selon son contexte social, politique et économique. Étant elle-même une ancienne militante dans la lutte contre le cancer du sein, Batt a eu recours à I'observation, aux entrevues et à une analyse documentaire approfondie pour cerner son sujet. L'auteure puise également dans les travaux d'anthropologues, de sociologues et d'historiens de la santé pour étayer son argumentaire. La principale contribution de l'ouvrage est de présenter une description historique dense et une analyse fine et nuancée de la problématique. Ce livre s'adresse à un public curieux, ayant un intérêt pour le sujet en question et touche notamment le lecteur pour qui les sciences sociales apportent un éclairage fondamental sur les questions d'ordre médical. Le présent résumé présente les principaux arguments de l'auteure qui supportent sa thèse, une description de la structure de l'ouvrage ainsi qu'une critique. 


\section{Description de la structure de l'ouvrage}

L'auteure a organisé son livre en deux parties principales. La première partie comprend les chapitres 1 et 2, qui s'articulent autour des caractéristiques du système public de soins de santé canadien, son évolution entre les années 1950 et 1980 et, surtout, les changements qui se sont opérés lors de l'adoption de politiques et de structures néolibérales. Le chapitre 2 met justement en contexte le développement des groupes associatifs au Canada et les politiques les entourant, notamment celles concernant leur financement. La deuxième partie comprend cinq chapitres traitant de trois thématiques. D'abord, la mise en place et le développement du mouvement contre le cancer du sein au Canada et le partenariat de ce mouvement avec l'industrie pharmaceutique - qu'elle nomme PHANGO. La thématique suivante est la description de débats éthiques et de contestations qu'a connu le milieu associatif du cancer du sein vis-à-vis son financement par I'industrie pharmaceutique. La troisième est l'analyse de l'auteure des rouages du partenariat entre les associations et I'industrie. La conclusion reprend les éléments de son analyse en soulignant comment s'opère le partenariat des PHANGO, comment celui-ci peut influencer les politiques de santé et celles reliées au secteur pharmaceutique, et comment les PHANGO arrivent à co-construire un savoir qui sert les intérêts des patients et de l'industrie.

\section{Arguments principaux}

Le partenariat entre l'industrie pharmaceutique et les associations de patientes qui luttent contre le cancer du sein s'est construit de façon graduelle et planifiée. Plusieurs évènements y ont contribué. D'abord, selon Batt, I'un des premiers facteurs est un ensemble de politiques néolibérales instaurées par le gouvernement fédéral qui ont progressivement restreint et coupé le financement de la part des instances gouvernementales et publiques que recevaient les associations de patients. En parallèle, on assiste également à une crise de confiance entre les différentes associations réparties à travers le Canada et les agences gouvernementales. L'industrie est donc, selon l'auteure, venue progressivement combler une demande financière ou logistique. Sur ce point, Batt est claire : les compagnies pharmaceutiques ont utilisé différentes stratégies, inspirées de celles utilisées avec les médecins, pour développer un partenariat avec les organisations de patientes. C'est ainsi que les différents acteurs ont bâti un agenda commun, pour promouvoir entre autres de nouveaux produits pharmaceutiques et améliorer l'accessibilité à de nouveaux traitements. De nombreuses conséquences ont découlé de ces alliances : notamment, avant d'être normalisées, elles ont fait l'objet de débats éthiques, de tensions et de résistances au sein des associations. Les différents discours présentés dans l'ouvrage montrent l'implantation d'une vision particulière de la science pharmaceutique, dans laquelle le travail des pharmaceutiques représente la réponse à la prise en charge du cancer du sein tout en étant responsable de l'innovation. Dans cette perspective, les médicaments, produits du marché libre, sont porteurs de personnalités et d'un message d'espoir pour les patientes. Pour conclure, selon Sharon Batt, les PHANGO ne servent pas les intérêts publics et perdent de leur légitimité à assurer un regard critique envers le travail de I'industrie et des produits de celle-ci.

C'est à travers les multiples exemples d'archives ou de propos retranscrits provenant d'entrevues avec plusieurs acteurs travaillant de près ou de loin pour la cause du cancer du sein que le lecteur perçoit le travail de recherche et de documentation qui supporte la vision de Sharon Batt. Elle parvient à apporter certaines nuances à la problématique, par exemple, en ce qui trait au développement et aux dynamiques internes des associations de différentes provinces canadiennes. Ceci nous permet de constater que les alliances entre associations de patientes et l'industrie, au Canada, n'est pas un mouvement homogène. Par ailleurs, et suite à l'effort considérable de l'auteure à vulgariser un nombre très important d'informations, la lecture de cet ouvrage requiert de rester attentif au risque de se perdre dans un dédale de renseignements. Qui plus est, Sharon Batt, en exposant les faits tissant les alliances entre les associations de patientes et l'industrie pharmaceutique, laisse son lecteur à ses propres interprétations.

\section{Références}

1.Batt s. Health Advocacy Inc; 2017. Vancouver: UBC Press.

Pour contacter l'auteur:

Stéphanie Collins

Candidate au doctorat

Université de Montréal

Faculté de pharmacie

C.P. 6128, succursale Centre-ville

Montréal, Québec, H3C 3J7

Canada

Courriel : stephanie.collins@umontreal.ca 